\section{CVIA}

REVIEW ARTICLE

pISSN 2508-707X / eISSN 2508-7088 https://doi.org/10.22468/cvia.2016.00045 CVIA 2017;1(1):13-22

CrossMark $\leftarrow$ click for updates

\title{
Cardiac CT and MRI for Assessment of Cardioembolic Stroke
}

\author{
Ye Ra Choi', Hack-Lyoung Kim², Hyung-Min Kwon³, \\ Eun Ju Chun ${ }^{4}$, Sung Min $\mathrm{Ko}^{5}$, Seung Min Yoo ${ }^{6}$, \\ Sang-Il Choi ${ }^{4}$, Kwang Nam Jin ${ }^{1}$ \\ 'Departments of Radiology, ${ }^{2}$ Cardiovascular Center, ${ }^{3}$ Neurology, Seoul Metropolitan \\ Government-Seoul National University Boramae Medical Center, Seoul, Korea \\ ${ }^{4}$ Department of Radiology, Seoul National University Bundang Hospital, Seongnam, Korea \\ ${ }^{5}$ Department of Radiology, Konkuk University Medical Center, Seoul, Korea \\ ${ }^{6}$ Department of Diagnostic Radiology, CHA University College of Medicine, Seongnam, Korea
}

Received: October 24, 2016

Revised: November 12, 2016

Accepted: November 14, 2016

Corresponding author

Kwang Nam Jin, MD

Department of Radiology,

Seoul Metropolitan Government-Seoul

National University Boramae Medical

Center, 20 Boramae-ro 5-gil,

Dongjak-gu, Seoul 07061, Korea

Tel: $82-2-870-2540$

Fax: 82-2-870-3539

E-mail: wlsrhkdska@gmail.com
Cardioembolic stroke is generally severe with a high risk for both early and long-term recurrence, requiring early diagnosis. Cardiac CT and MRI are accurate noninvasive alternatives to echocardiography which can demonstrate the potential cardiac source of an embolism and exclude other potential sources. The major sources of cardioembolic stroke include atrial fibrillation, recent myocardial infarction, valvular heart disease, endocarditis, and atrial myxoma. Minor sources of cardioembolism are paradoxical embolism due to patent foramen ovale and atrial septal aneurysm, atrial or ventricular septal defects, calcific aortic stenosis, and mitral annular calcification. The appropriate clinical application of cardiac CT and MRI can allow an exact and timely diagnosis to be made in embolic stroke patients.

Key words Intracranial embolism · Stroke · EKG-triggered imaging techniques . Tomography, spiral computed · Magnetic resonance imaging.

\section{INTRODUCTION}

Cardioembolic stroke accounts for $14-30 \%$ of all ischemic stokes and is generally severe with a high risk of early and longterm recurrence, and high mortality [1]. Therefore, early diagnosis of cardioembolic stroke is critical so that anticoagulation therapy can be initiated for secondary prevention. Variable cardiac disorders have been proposed as potential sources of embolism. The major sources of cardioembolic stroke include atrial fibrillation, recent myocardial infarction, valvular heart disease, endocarditis, and atrial myxoma. Major sources carry a relatively high risk of initial and recurrent stroke and are convincingly linked to a cardioembolic mechanism [2]. Minor sources are paradoxical embolism due to patent foramen ovale (PFO) and atrial septal aneurysm (ASA), atrial or ventricular septal defects, calcific aortic stenosis, and mitral annular calcification (MAC) [2]. Minor sources are common in the general population, and these conditions have a low or uncertain associated risk of initial and recurrent stroke [2]. Imaging of potential sources allows the identification of the potential cardiac embo-

(a) This is an Open Access article distributed under the terms of the Creative Commons Attribution Non-Commercial License (http://creativecommons.org/licenses/by$\mathrm{nc} / 3.0$ ) which permits unrestricted non-commercial use, distribution, and reproduction in any medium, provided the original work is properly cited. lism source and can exclude other potential sources of cerebral ischemia. Given the noninvasive nature and wide availability of transthoracic echocardiography (TTE), it is one of the initial cardiac imaging tools used for diagnosis of potential cardioembolic sources. Transesophageal echocardiography (TEE) provides more detail of the atria and aorta than TTE, although it is more invasive and requires more training to be performed adequately [3]. Cardiac CT or MRI can be accurate noninvasive alternatives to TEE or TTE in the following circumstances: 1) patients who cannot tolerate TEE, 2) questionable results from TTE for the detection of thrombus in the left ventricle, 3 ) inconclusive results of TEE, or 4) characterization of a cardiac mass detected on TTE or TTE (Table 1). The aim of this review is to present recent investigations on using cardiac CT or MRI in cardioembolic stroke cases.

\section{ATRIAL FIBRILLATION}

Atrial fibrillation is the most common cause of embolic sources, and about $60 \%$ of cardioembolic strokes are caused by left atrium (LA) and left atrial appendage (LAA) thrombi secondary to atrial fibrillation or flutter [4]. Atrial fibrillation causes strokes because of inadequate LA or LAA contraction 


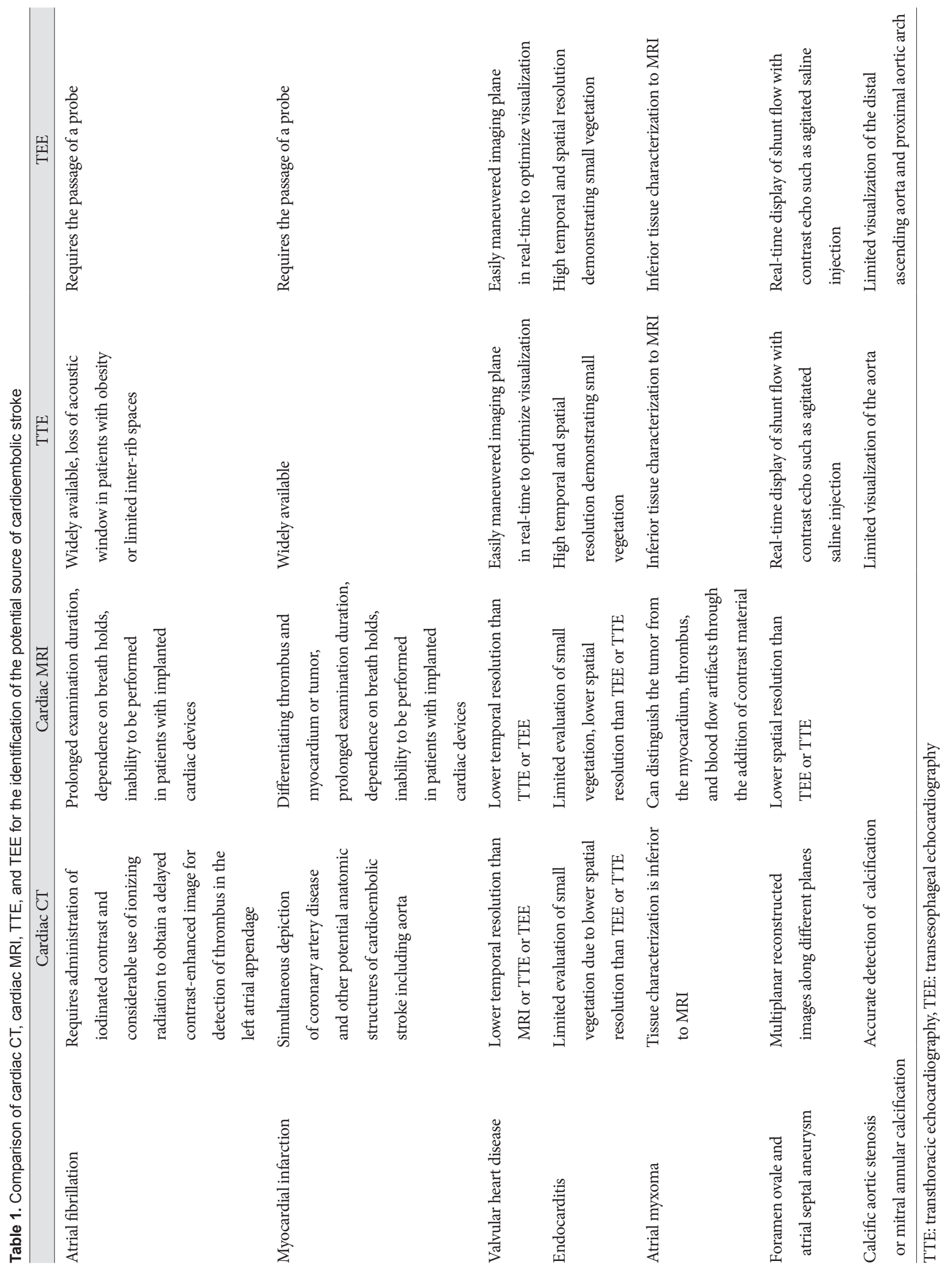



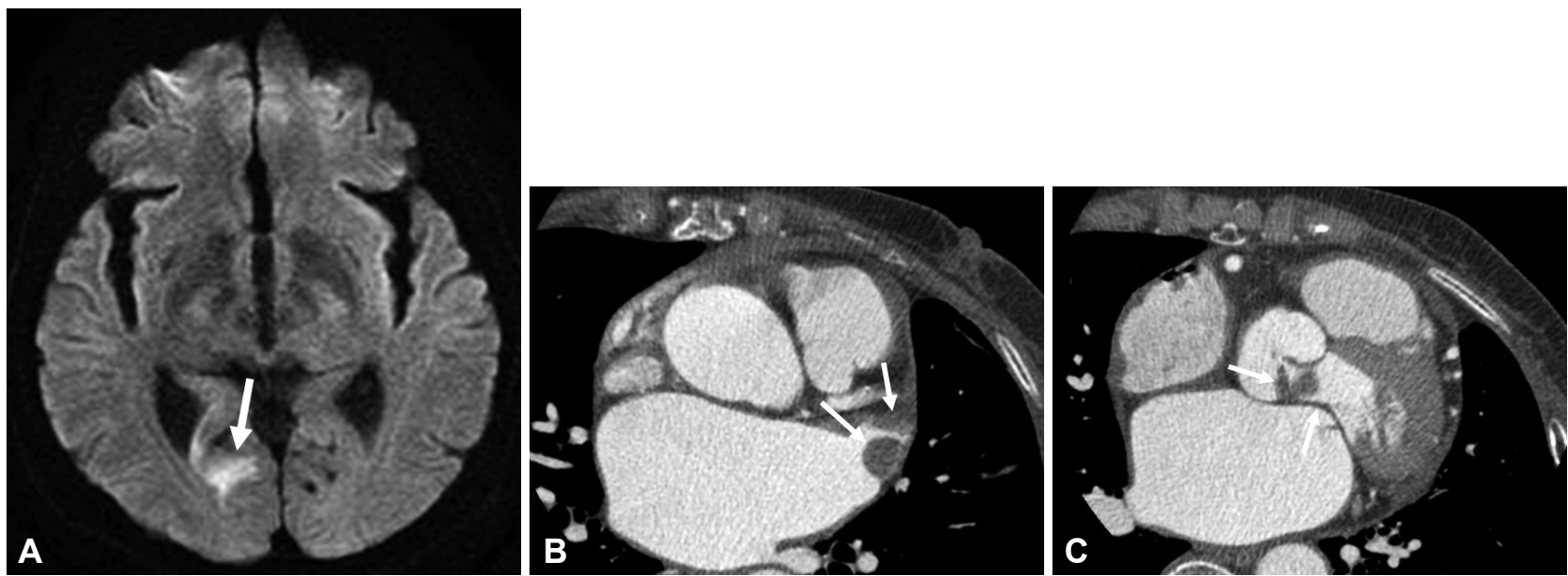

Fig. 1. A left atrial appendage thrombus in a 67-year-old woman. (A) A diffusion weighed brain MR image shows a small area of high signal intensity (arrow) in the right occipital lobe suggesting an acute cerebral infarction. (B) Cardiac CT shows a mural thrombus (arrows) in the left atrial appendage. (C) Cardiac CT also demonstrates aortic and mitral valvular thickening (arrows) suggesting rheumatic valvular disease.
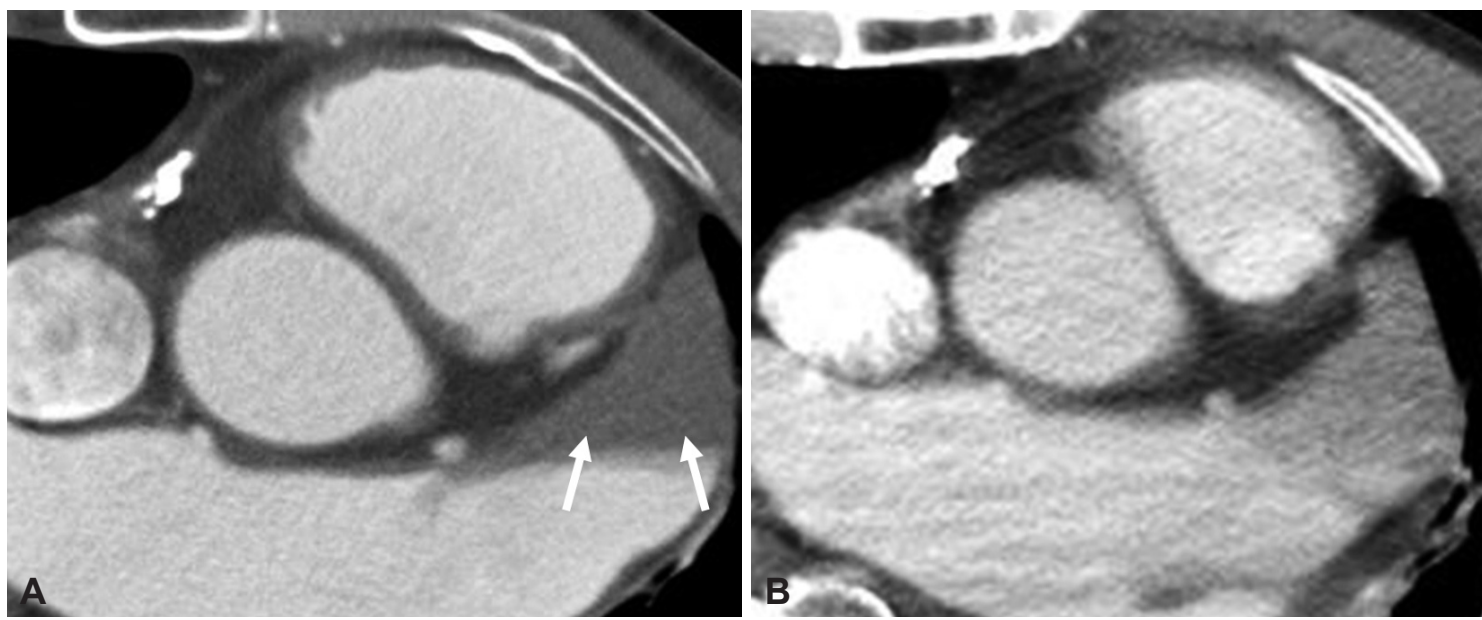

Fig. 2. Circulatory stasis in the left atrial appendage in a 78-year-old man. (A) Cardiac CT shows a filling defect in the left atrial appendage (arrows). (B) Delayed image acquisition reveals contrast enhancement in the left atrial appendage, differentiating circulatory stasis from thrombi.
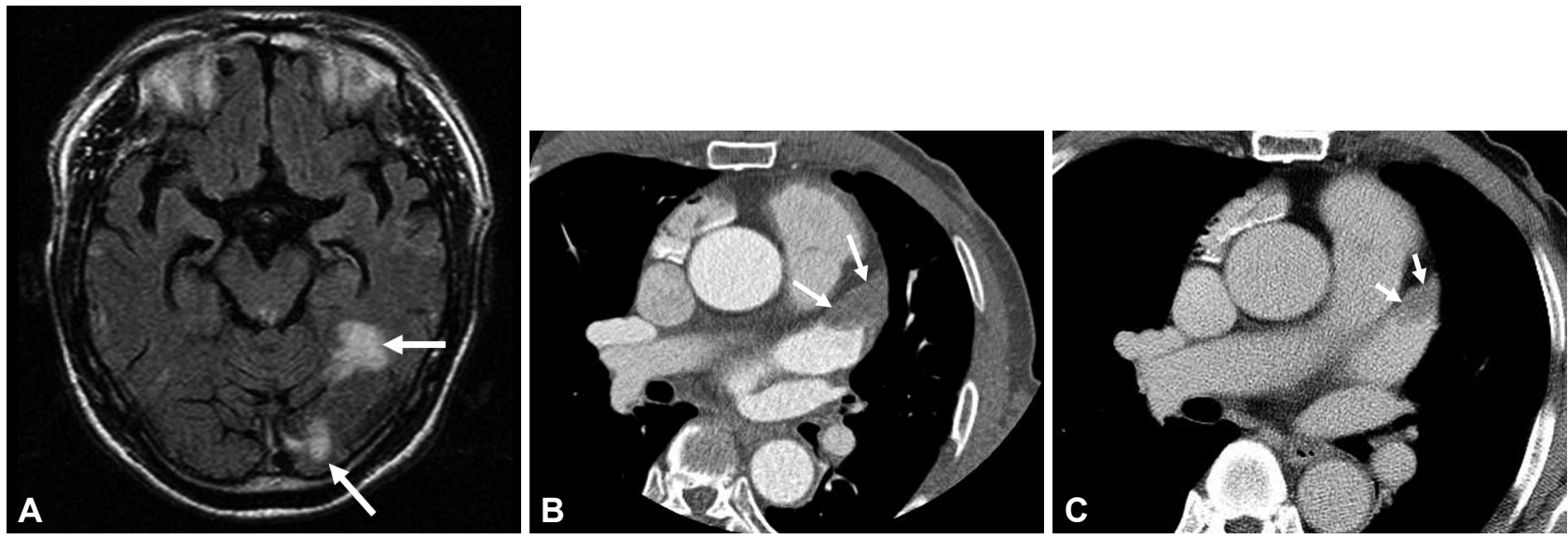

Fig. 3. A left atrial appendage thrombus in a 73-year-old man. (A) A diffusion weighed brain MR image shows small areas of high signal intensity (arrows) in the left occipital lobe suggesting an acute cerebral infarction. (B) Cardiac CT shows a filling defect (arrows) in the left atrial appendage. (C) Delayed image acquisition reveals a persistent nodular filling defect in the left atrial appendage (short arrows) suggesting a thrombus. 
which leads to stasis that predisposes thrombus formation, and consequently an increased rate for cerebral embolization [2]. TEE is usually chosen for examination of LAA thrombi due to its high sensitivity and specificity for the detection of thrombi [5]. However, TEE is a semi-invasive test, and may not be adequate in various circumstances including multilobed appendages, large pectinate muscles, and dense spontaneous echo contrast, which can obscure a thrombus in the LAA [6].

With recent improvements in temporal and spatial resolution, cardiac CT has emerged as an acceptable alternative for evaluating an intracardiac thrombus, which is observed as a filling defect (Fig. 1) [7]. Delayed scanning has been reported to improve the specificity and positive predictive value of cardiac
CT for the detection of LAA thrombi (Figs. 2 and 3). In a recent meta-analysis, the sensitivity and specificity of cardiac CT was 0.957 (95\% confidence interval: 0.928-0.977) and 0.917 (95\% confidence interval: 0.905-0.927), respectively [8]. This study showed that diagnostic accuracy improved significantly with delayed imaging, ECG gating, and heart rate control with a sensitivity of 0.991 (95\% confidence interval: $0.951-1.000)$ and specificity of 0.989 (95\% confidence interval: 0.977-0.995). Dual-energy cardiac CT can also be used for differentiating LAA thrombi from circulatory stasis with high sensitivity [7]. Regarding delayed phase scanning, contrast enhancement of the first contrast materials in the LAA results in a different attenuation between a thrombus and circulatory stasis. A recent study
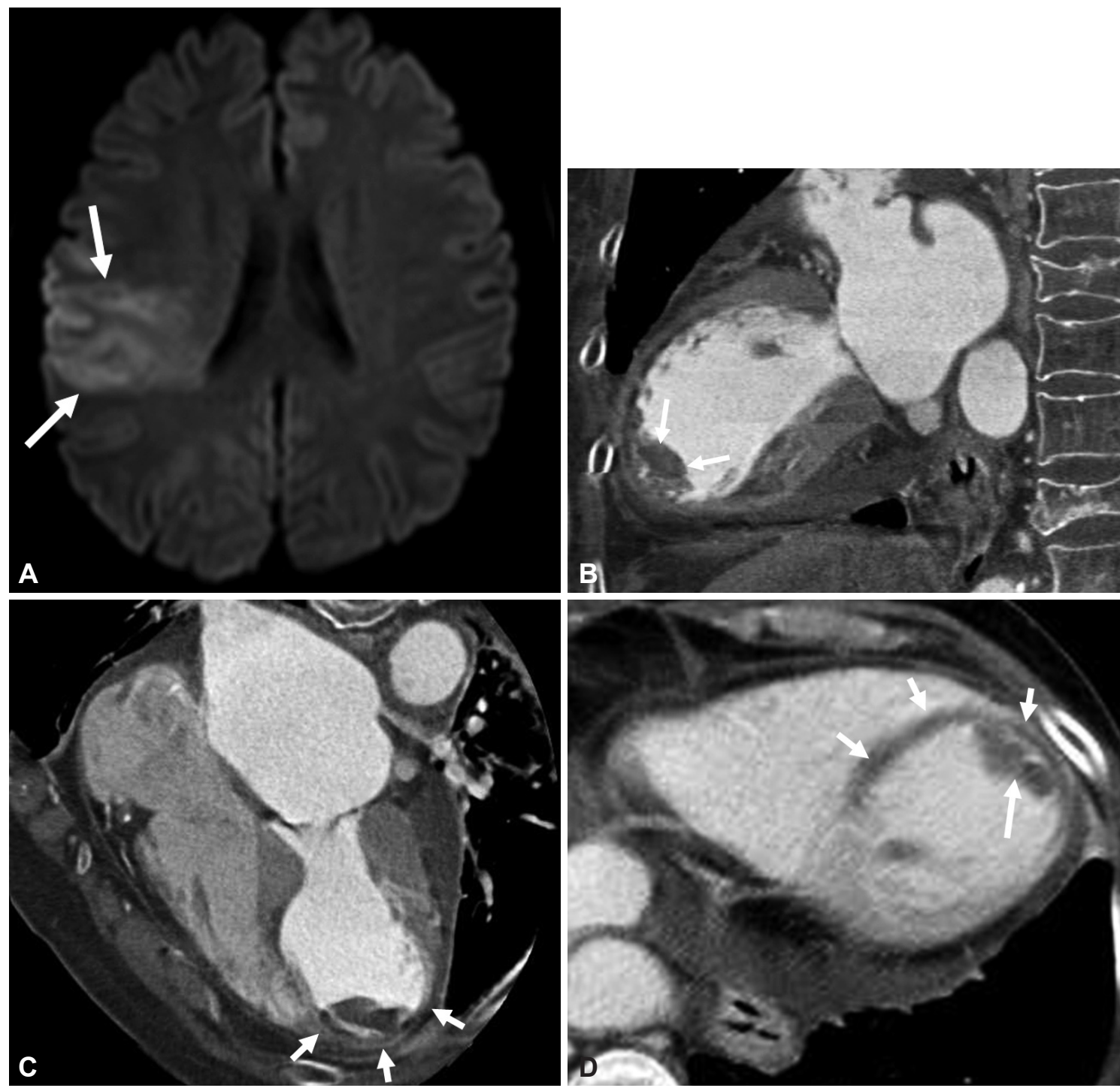

Fig. 4. A left ventricular thrombus in a 36-year-old man with a history of acute myocardial infarction two weeks prior. (A) A diffusion weighed brain MR image shows high signal intensity (arrow) in the right frontal lobe precentral gyrus suggesting acute cerebral infarction. (B) A two chamber view image of cardiac CT shows a nodular filling defect (arrows) in the left ventricular apex surrounded by contrast material. (C) Myocardial thinning and aneurysm (short arrows) in the left ventricle apex on the four chamber view image suggest a recent myocardial infarction. (D) Delayed image acquisition demonstrates a perfusion defect (short arrows) in the left ventricular apex and apical segment with non-enhancement of thrombus in the apex (arrow). 
revealed that a cardiac CT measurement with a LAA/aorta Hounsfield units (HUs) ratio $<0.245$ in the arterial and venous phases can be a quantitative indicator for the presence of a thrombus [9], whereas another study concluded that additional calculation of the HU ratio did not improve the diagnostic performance of cardiac CT for detecting LAA thrombi [10]. Because a two-phase protocol increases patient radiation exposure, the supplementary role of CT can be most valuable in cases where performing TTE or TEE is hampered by limited experience or contraindication due to medical reasons [11]. Cardiac MRI can be proposed as an effective, safer, and comprehensive imaging modality comparable to TEE for the evaluation of LAA thrombi, although its slower image acquisition and inability to be used in patients with implanted metallic devices interfere with the feasibility of cardiac MRI [12].

\section{RECENT MYOCARDIAL INFARCTION}

Approximately $1.0-2.5 \%$ of patients with acute myocardial infarction experience a stroke during the first 4 weeks after the infarction [13]. Factors that increase the risk of stroke are severe left ventricular (LV) dysfunction with low cardiac output and LV aneurysm or thrombus. The incidence of LV mural thrombus remains high (15-40\%) after anterior infarction [14]. The incidence of early embolism is also high (up to $22 \%$ ) in the presence of a mural thrombus that is mobile or protrudes into
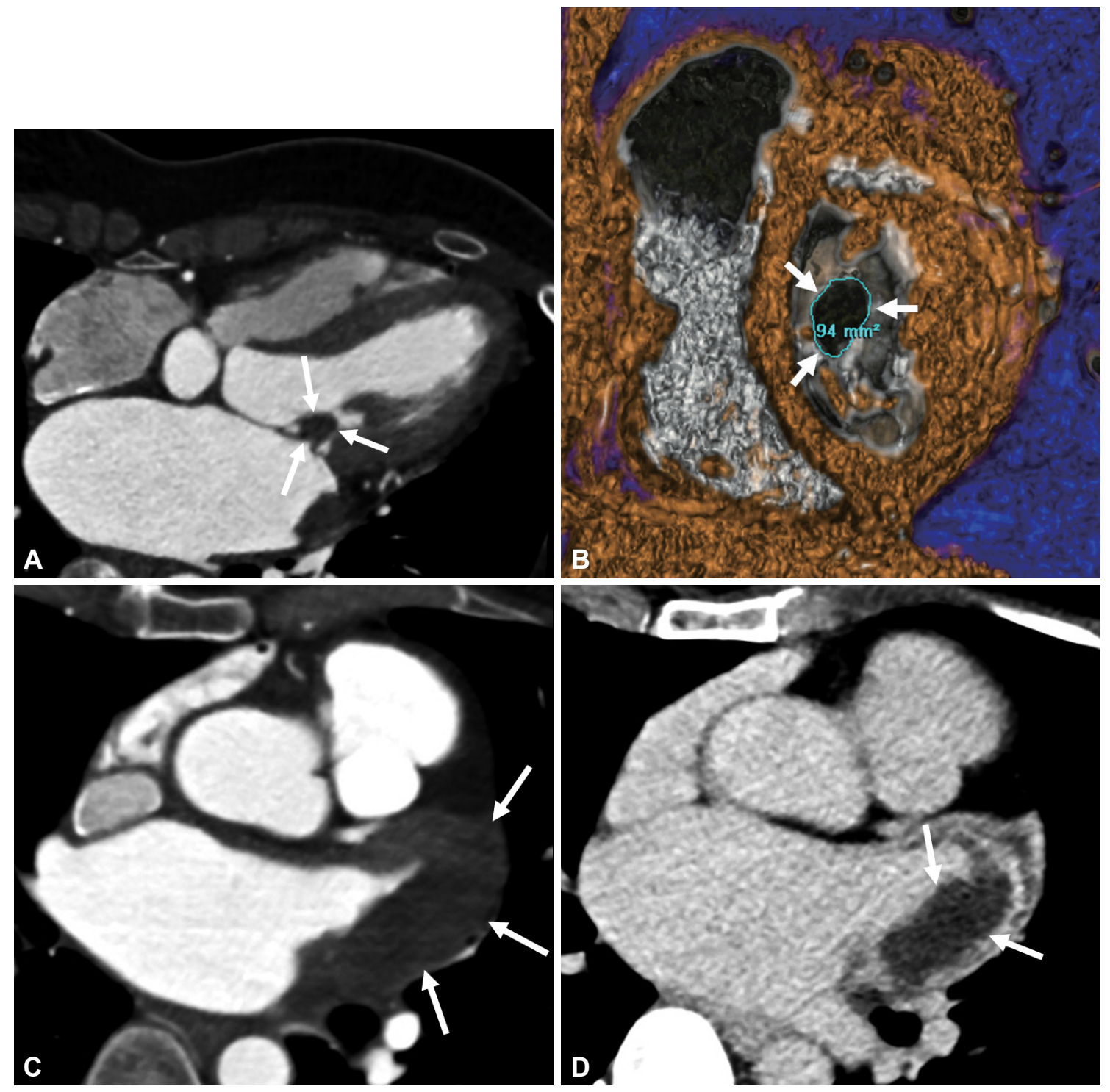

Fig. 5. A left atrial thrombus in a 46-year-old woman with mitral stenosis. (A) Cardiac CT shows a thickened leaflet and diastolic bowing of the leaflets in a hockey-stick shape deformity (arrows). (B) A volume rendering reformatted image shows the mitral valvular area measurement (short arrows). (C) Cardiac CT demonstrates a diffuse mural thrombus in the left atrium (arrows) and (D) persistent nodular filling defect in the left atrial appendage (arrows) on the delayed image acquisition. 
the ventricle [2]. Cardiac CT and MRI are both excellent tools to evaluate a LV thrombus. On cardiac CT, a thrombus is observed as a low-attenuation area surrounded by an infarcted myocardium (Fig. 4). In a study on 31 patients by Bittencourt et al. [15], CT attenuation within LV thrombi was significantly lower than myocardial attenuation, and a threshold of $65 \mathrm{HU}$ yielded $94 \%$ sensitivity and $97 \%$ specificity to differentiate a thrombus from the myocardial wall. Contrast-enhanced cardiac MRI is not only an excellent technique to depict myocardial in- farction and scar tissue, but also a sensitive method for detecting a LV thrombus [16]. Delayed enhancement cardiac MRI using gadolinium contrast differentiates a thrombus from the surrounding myocardium as a thrombus is avascular and thus characterized by the absence of contrast uptake. Srichai et al. [17] reported that contrast-enhanced cardiac MRI, incorporating delayed enhancement cardiac MRI and cine-cardiac MRI $(n=160)$, showed the highest sensitivity and specificity for thrombus detection ( $88 \%$ and $99 \%$, respectively) compared to
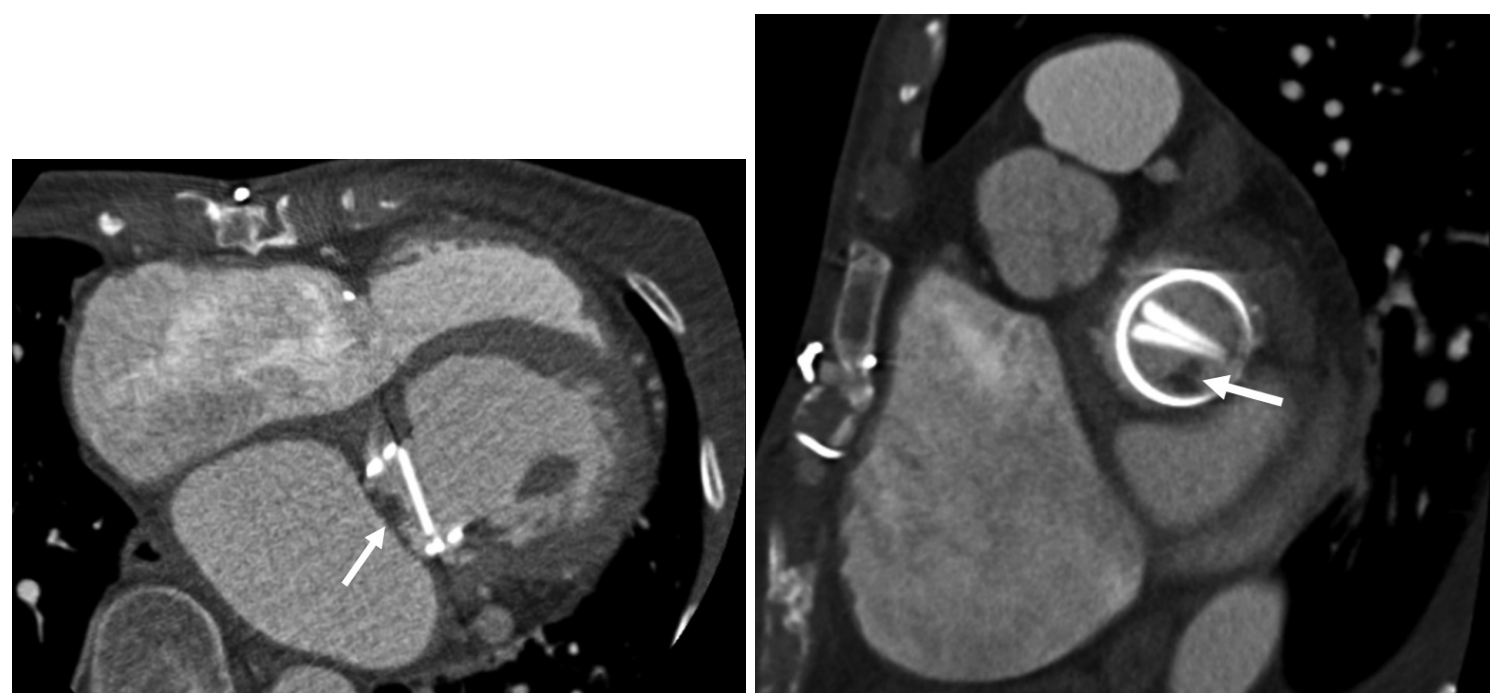

Fig. 6. Infective endocarditis in a 41-year-old woman who underwent prosthetic mitral valve replacement. Cardiac CT shows a small low attenuating nodule attached to the prosthetic mitral valve suggesting vegetation (arrows). Cardiac surgeries revealed a pannus and abscess around the prosthetic mitral valve.
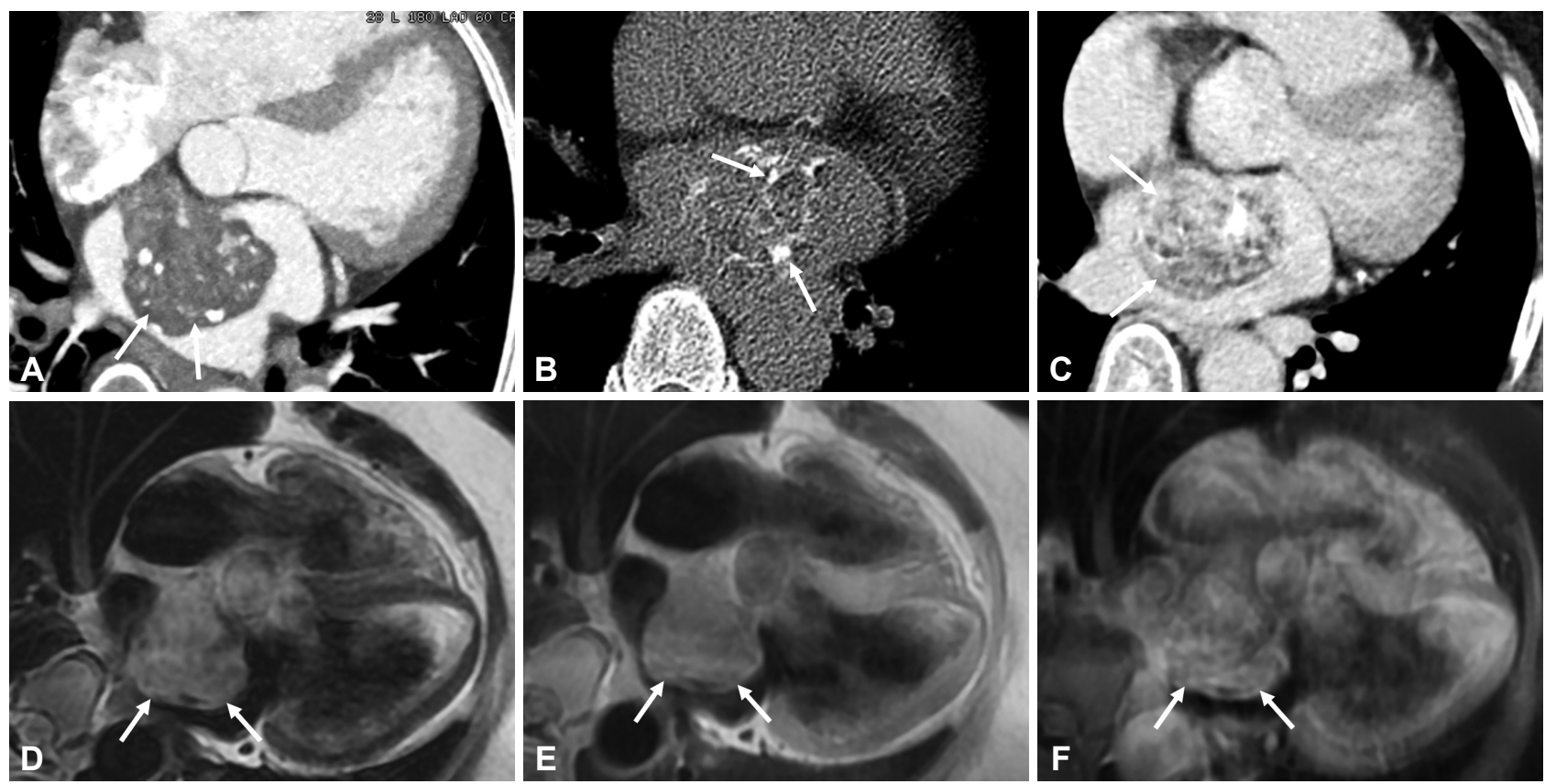

Fig. 7. Cardiac myxoma in a 60-year-old woman. (A and B) Cardiac CT shows about a $5 \mathrm{~cm}$ sized left atrial intraluminal protruding mass attached to the interatrial septum with multiple nodular calcifications (arrows). (C) Delayed image acquisition demonstrates diffuse enhancement in the mass (arrows). Cardiac MRI demonstrates high signal intensity in the mass (arrows) on both T1 (D) and T2 (E) weighed images and gadolinium enhancement $(F)$. 
TTE (23\% and 96\%, respectively) and TEE (40\% and 96\%, respectively). In a study by Weinsaft et al. [16], among 784 patients with systolic dysfunction, delayed enhancement cardiac MRI detected a thrombus in 55 patients (7.0\%) and cine-cardiac MRI in 37 patients (4.7\%), $\mathrm{p}<0.005$.

Rheumatic valvular heart disease and mechanical prosthetic valves are well-known risk factors for cardioembolic stroke [2]. The two most common valve abnormalities are mitral stenosis and calcific aortic stenosis. Systemic embolism occurs in 9-14\% of patients with mitral stenosis and may be the initial presentation of mitral stenosis [18]. The risk of stroke in mitral stenosis is amplified in the presence of atrial fibrillation. Valvular atrial fibrillation is commonly accompanied by mitral stenosis, requiring anticoagulation treatment. Cardiac CT can demonstrate a thickened leaflet and diastolic bowing of the leaflets in a hockey-stick shape deformity as well as calcification in the mitral valve leaflet or commissure in patients with mitral stenosis (Fig. 5) [19]. Geometric measurement of the mitral orifice area on car- diac CT is well correlated with TEE [20]. Cardiac CT demonstrates valvular vegetation as an oval or round filling defect on the mitral or aortic valve [21]. Because small vegetation can be disregarded as mere valvular thickening on cardiac CT, careful interpretation is needed to avoid false-negative results [11].

\section{ENDOCARDITIS}

Systemic embolization occurs in $22-50 \%$ of infective endocarditis cases [22]. Although TEE has great sensitivity in the detection of vegetations, cardiac CT can be used as an alternative tool for the assessment of endocarditis (Fig. 6) [23]. In a recent study by Feuchtner et al. [23], the diagnostic performance of cardiac CT for the detection of valvular abnormalities related to infective endocarditis was compared with TEE. Cardiac CT had a sensitivity of $97 \%$ (95\% confidence interval: $82-100 \%)$ and specificity of $88 \%$ (95\% confidence interval: $47-100 \%$ ) on a perpatient basis. In their study, cardiac CT correctly identified 26
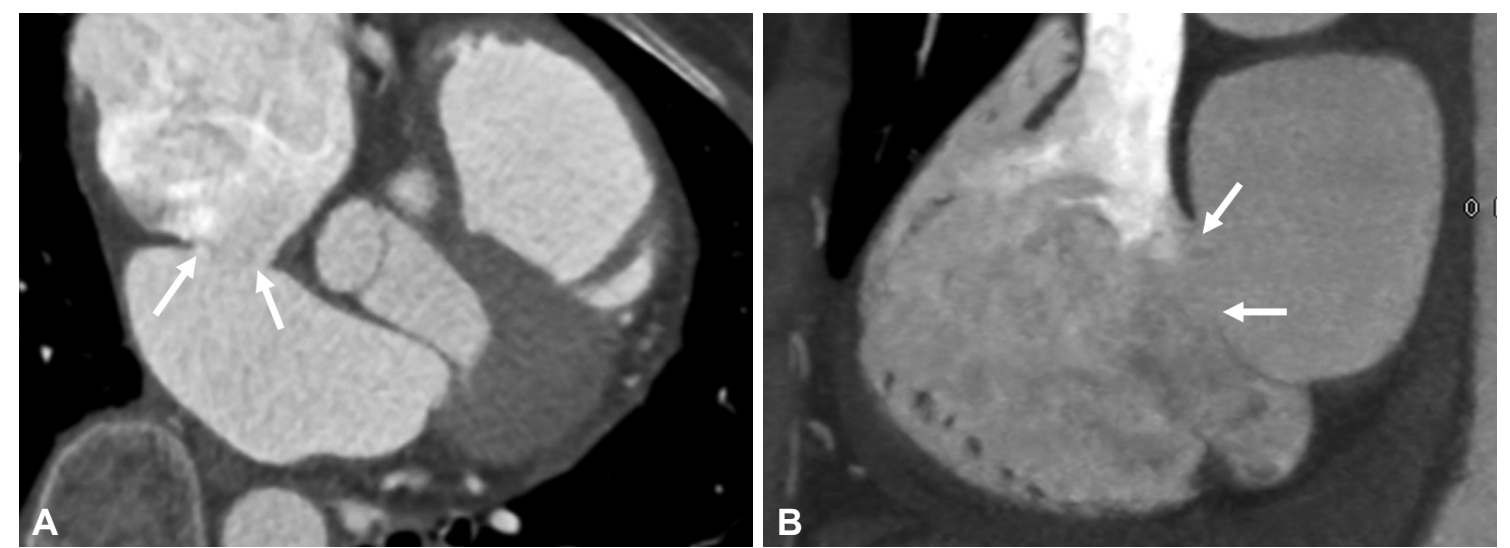

Fig. 8. An atrial septal defect in a 57-year-old woman. A reformatted image (A) and short-axis image (B) of cardiac CT show a $1.8 \mathrm{~cm}$ sized defect in the interatrial septum suggesting a secondum type atrial septal defect (arrows).
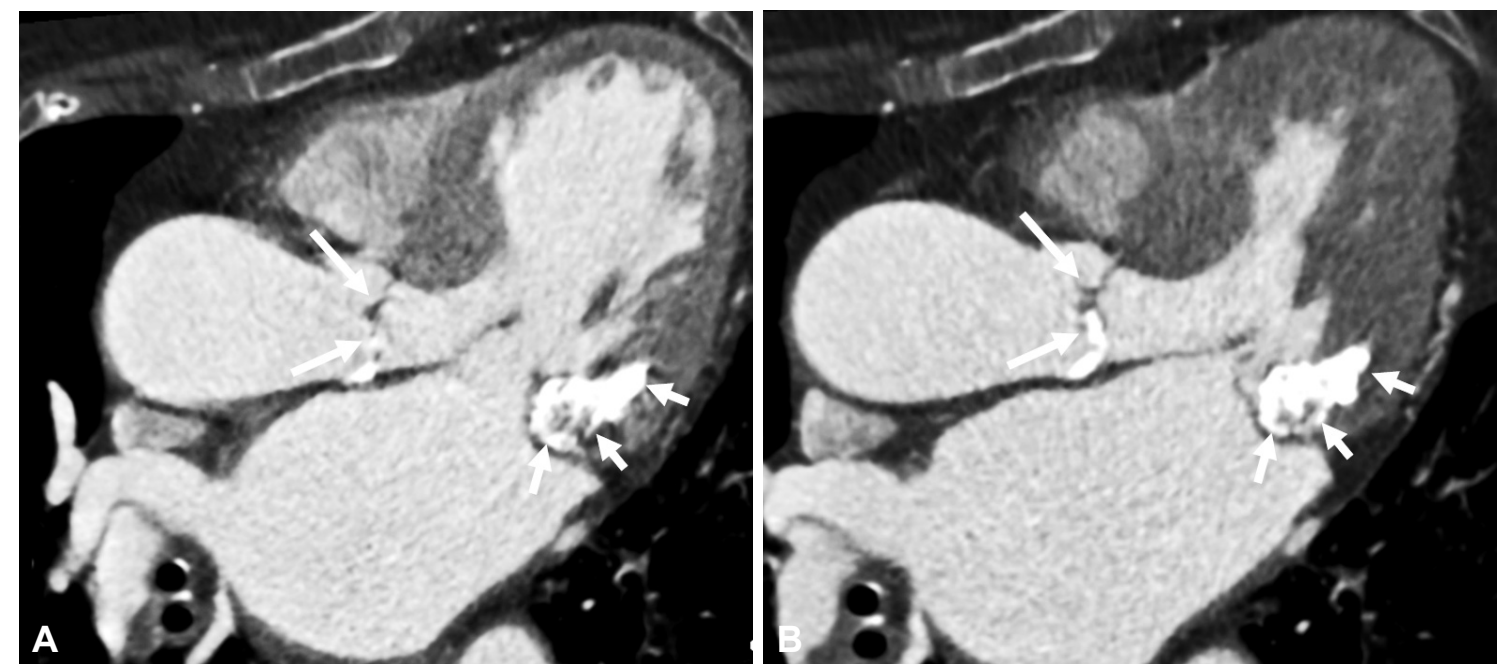

Fig. 9. A mitral annular calcification in an 83-year-old woman. Cardiac CT in the diastolic (A) and systolic phase (B) shows an extensively calcified mitral annulus (short arrows) with left atrial dilatation and severe aortic stenosis (arrows). 
of 27 (96\%) patients with valvular vegetations and 9 of 9 (100\%) patients with abscesses/pseudoaneurysms compared to the intraoperative specimen. Regarding the lower temporal resolution of cardiac CT as compared to echocardiography, cardiac CT can be a complementary imaging modality by providing more accurate anatomic information regarding the perivalvular extent of abscesses/pseudoaneurysms than TEE [24] and allows concurrent imaging of coronary arteries prior to valve replacement surgery [25].

\section{ATRIAL MYXOMA}

Cardiac tumors such as myxomas and papillary fibroelastomas are associated with a high frequency of embolic events. Myxomas occur in the LA in $75 \%$ of cases, often originating from the fossa ovalis area of the interatrial septum [26]. Myxomas tend to embolize to the systemic circulation in 30 to $40 \%$ of cases, and to the cerebral circulation in over $50 \%$ of cases. Embolization may be caused by tumor fragmentation, or be secondary to superimposed thrombus formation. Although TEE is the mainstay for the clinical diagnosis of cardiac mass, cardiac MRI is the reference imaging technique for further differentiation and characterization of cardiac tumors. In addition to cine imaging and steady-state free precession, black blood imaging, fat-suppressed sequences, perfusion, and late gadolinium enhancement are used as MR protocols [4]. Cardiac CT is also an excellent method for assessment of a mass due to its higher spatial resolution and its ability to evaluate calcification, fat, thrombi, and fibrous material (Fig. 7). In some cases, an atrial thrombus may have pedunculation and can be misdiagnosed as a myxoma, which can lead to an unnecessary surgical resection [27]. According to a cardiac CT study by Scheffel et al. [28], myxomas are significantly larger than thrombi, most often originate from the fossa ovalis, and are predominantly polypoid, with a minority having a villous shape [26], whereas thrombi are smaller than myxomas and most often originate from the appendage. It is reported that villous-shaped myxomas and mobile polypoid thrombi are highly associated with embolic strokes [29]. Regarding differentiating between benign and malignant intracardiac masses, cardiac MRI is excellent due to its multiplanar capabilities and soft tissue characterization. For example, lipomatous hypertrophy of the interatrial septum, which is a common benign variant mimicking mass, can be correctly identified using cardiac MRI [30].

\section{PARADOXICAL EMBOLI}

PFO is present in a third of stroke patients, and is found in up to $40 \%$ of patients with ischemic stroke younger than 55 years of age [31]. The association between PFO and cryptogenic stokes has been well established [32]. It is frequently considered as a possible cardioembolic source since it allows for potential paradoxical embolism, a systemic arterial embolus from a venous source [3]. PFO and atrial septal defect (ASD) can be differentiated based on the direction of the contrast jet from the left to right atrium and the appearance of the atrial septum on cardiac CT [33]. A contrast jet flowing toward the inferior vena cava with the channel-like appearance of atrial septum can confirm a PFO. On the other hand, a contrast jet flowing perpendicular to the atrial septum with the appearance of a membrane with a hole is an ASD (Fig. 8). An ASA can cause a stroke by paradoxical embolism of a thrombus originating in the aneurysm or by inducing supraventricular arrhythmia. ASA is defined as the presence of interatrial septal bulging with an excursion of $>1.0 \mathrm{~cm}$ with $>1.5 \mathrm{~cm}$ of base span [21]. Paradoxical embolism, supraventricular arrhythmia, and thrombus from a coexistent ASA are possible mechanisms of stroke in PFO. Patients with PFO and ASAs had an increased risk ratio (4.2) for recurrent stroke [34].

\section{CALCIFIC AORTIC STENOSIS AND MITRAL VALVE CALCIFICATION}

Aortic stenosis is a rare cause of stroke which is usually calcific. Large emboli in patients with calcific aortic stenosis have been associated with cardiac catheterization and balloon valvuloplasty [35]. Regarding cardiac CT imaging in aortic stenosis, the measurement value for the aortic valve area is larger than the echocardiographic result [36] and valvular calcification adversely affects the accuracy of aortic valve area measurement [37]. MAC is also a possible source of cerebral embolism with a 2.1 increase in the relative risk of stroke, and a fivefold increase in the risk of stroke in the presence of atrial fibrillation [1]. $\mathrm{MAC}$ is chronic degeneration of the mitral valve fibrous ring. In patients with suspected embolic stroke of uncertain etiology, dense MAC is an important marker for aortic atherosclerosis associated with a high risk of embolism [38]. On cardiac CT, MAC can be defined as calcification located at the junction between the LA and LV (Fig. 9). When liquefaction necrosis develops in the calcification, it can look like the left atrial mass in the posterior mitral annulus on TTE, mimicking a left atrial fibroma [39]. With the use of cardiac CT or MRI, MAC can be accurately diagnosed by revealing areas of calcifications in the mass along with their exact locations [40].

\section{CONCLUSION}

Cardioembolic stroke can arise from various cardiac disorders. Appropriate clinical application of cardiac CT or MRI can be useful to quickly identify or exclude the cardiac source and 
make an exact diagnosis in patients with embolic stroke.

\section{Conflicts of Interest}

The authors declare that they have no conflict of interest.

\section{REFERENCES}

1. Di Tullio MR, Homma S. Mechanisms of cardioembolic stroke. Curr Cardiol Rep 2002;4:141-148.

2. Arboix A, Alió J. Cardioembolic stroke: clinical features, specific cardiac disorders and prognosis. Curr Cardiol Rev 2010;6:150-161.

3. Wessler BS, Kent DM. Controversies in cardioembolic stroke. Curr Treat Options Cardiovasc Med 2015;17:358.

4. Pagán RJ, Parikh PP, Mergo PJ, Gerber TC, Mankad R, Freeman WD, et al. Emerging role of cardiovascular CT and MRI in the evaluation of stroke. AJR Am J Roentgenol 2015;204:269-280.

5. Budoff MJ, Shittu A, Hacioglu Y, Gang E, Li D, Bhatia H, et al. Comparison of transesophageal echocardiography versus computed tomography for detection of left atrial appendage filling defect (thrombus). Am J Cardiol 2014;113:173-177.

6. Hur J, Kim YJ, Lee HJ, Nam JE, Ha JW, Heo JH, et al. Dual-enhanced cardiac CT for detection of left atrial appendage thrombus in patients with stroke: a prospective comparison study with transesophageal echocardiography. Stroke 2011;42:2471-2477.

7. Hur J, Kim YJ, Lee HJ, Nam JE, Hong YJ, Kim HY, et al. Cardioembolic stroke: dual-energy cardiac CT for differentiation of left atrial appendage thrombus and circulatory stasis. Radiology 2012;263:688-695.

8. Zou H, Zhang Y, Tong J, Liu Z. Multidetector computed tomography for detecting left atrial/left atrial appendage thrombus: a meta-analysis. Intern Med J 2015;45:1044-1053.

9. Taina M, Vanninen R, Sipola P, Muuronen A, Jäkälä P, Hedman M. Cardiac CT Differentiates Left Atrial Appendage Thrombi from Circulatory Stasis in Acute Stroke Patients. In Vivo 2016;30:671-676.

10. Homsi R, Nath B, Luetkens JA, Schwab JO, Schild HH, Naehle CP. Can contrast-enhanced multi-detector computed tomography replace transesophageal echocardiography for the detection of thrombogenic milieu and thrombi in the left atrial appendage: a prospective study with 124 patients. Rofo 2016;188:45-52.

11. Ajlan AM, Bagdadi RR, Alama MN, Ayoub O. Impact of implementing cardiac CT in evaluating patients suspected of cardioembolic stroke. J Comput Assist Tomogr 2016;40:380-386.

12. Rathi VK, Reddy ST, Anreddy S, Belden W, Yamrozik JA, Williams RB, et al. Contrast-enhanced CMR is equally effective as TEE in the evaluation of left atrial appendage thrombus in patients with atrial fibrillation undergoing pulmonary vein isolation procedure. Heart Rhythm 2013;10:10211027.

13. Mooe T, Eriksson P, Stegmayr B. Ischemic stroke after acute myocardial infarction. A population-based study. Stroke 1997;28:762-767.

14. Solheim S, Seljeflot I, Lunde K, Bjørnerheim R, Aakhus S, Forfang K, et al. Frequency of left ventricular thrombus in patients with anterior wall acute myocardial infarction treated with percutaneous coronary intervention and dual antiplatelet therapy. Am J Cardiol 2010;106:1197-1200.

15. Bittencourt MS, Achenbach S, Marwan M, Seltmann M, Muschiol G, Ropers $\mathrm{D}$, et al. Left ventricular thrombus attenuation characterization in cardiac computed tomography angiography. J Cardiovasc Comput Tomogr 2012;6:121-126.

16. Weinsaft JW, Kim HW, Shah DJ, Klem I, Crowley AL, Brosnan R, et al. Detection of left ventricular thrombus by delayed-enhancement cardiovascular magnetic resonance prevalence and markers in patients with systolic dysfunction. J Am Coll Cardiol 2008;52:148-157.

17. Srichai MB, Junor C, Rodriguez LL, Stillman AE, Grimm RA, Lieber ML, et al. Clinical, imaging, and pathological characteristics of left ventricular thrombus: a comparison of contrast-enhanced magnetic resonance imaging, transthoracic echocardiography, and transesophageal echocar- diography with surgical or pathological validation. Am Heart J 2006;152: 75-84.

18. Weir NU. An update on cardioembolic stroke. Postgrad Med J 2008;84: 133-142; quiz 139-140.

19. Rajani R, Khattar R, Chiribiri A, Victor K, Chambers J. Multimodality imaging of heart valve disease. [Article in English, Portuguese] Arq Bras Cardiol 2014;103:251-263.

20. Messika-Zeitoun D, Serfaty JM, Laissy JP, Berhili M, Brochet E, Iung B, et al. Assessment of the mitral valve area in patients with mitral stenosis by multislice computed tomography. J Am Coll Cardiol 2006;48:411-413.

21. Lee K, Hur J, Hong SR, Suh YJ, Im DJ, Kim YJ, et al. Predictors of recurrent stroke in patients with ischemic stroke: comparison study between transesophageal echocardiography and cardiac CT. Radiology 2015;276: 381-389.

22. Baddour LM, Wilson WR, Bayer AS, Fowler VG Jr, Bolger AF, Levison $\mathrm{ME}$, et al. Infective endocarditis: diagnosis, antimicrobial therapy, and management of complications: a statement for healthcare professionals from the Committee on Rheumatic Fever, Endocarditis, and Kawasaki Disease, Council on Cardiovascular Disease in the Young, and the Councils on Clinical Cardiology, Stroke, and Cardiovascular Surgery and Anesthesia, American Heart Association: endorsed by the Infectious Diseases Society of America. Circulation 2005;111:e394-e434.

23. Feuchtner GM, Stolzmann P, Dichtl W, Schertler T, Bonatti J, Scheffel H, et al. Multislice computed tomography in infective endocarditis: comparison with transesophageal echocardiography and intraoperative findings. J Am Coll Cardiol 2009;53:436-444.

24. Kim RJ, Weinsaft JW, Callister TQ, Min JK. Evaluation of prosthetic valve endocarditis by 64-row multidetector computed tomography. Int J Cardiol 2007;120:e27-e29.

25. Meijboom WB, Mollet NR, Van Mieghem CA, Kluin J, Weustink AC, Pugliese F, et al. Pre-operative computed tomography coronary angiography to detect significant coronary artery disease in patients referred for cardiac valve surgery. J Am Coll Cardiol 2006;48:1658-1665.

26. Reynen K. Cardiac myxomas. N Engl J Med 1995;333:1610-1617.

27. Hesse B, Murphy RT, Myles J, Huang J, Sabik EM. Images in cardiovascular medicine. A left atrial appendage thrombus mimicking atrial myxoma. Circulation 2006;113:e456-e457.

28. Scheffel H, Baumueller S, Stolzmann P, Leschka S, Plass A, Alkadhi H, et al. Atrial myxomas and thrombi: comparison of imaging features on CT. AJR Am J Roentgenol 2009;192:639-645.

29. Burke A, Jeudy J Jr, Virmani R. Cardiac tumours: an update: cardiac tumours. Heart 2008;94:117-123.

30. Tumma R, Dong W, Wang J, Litt H, Han Y. Evaluation of cardiac masses by CMR-strengths and pitfalls: a tertiary center experience. Int J Cardiovasc Imaging 2016;32:913-920.

31. Cabanes L, Mas JL, Cohen A, Amarenco P, Cabanes PA, Oubary P, et al. Atrial septal aneurysm and patent foramen ovale as risk factors for cryptogenic stroke in patients less than 55 years of age. A study using transesophageal echocardiography. Stroke 1993;24:1865-1873.

32. Hari P, Pai RG, Varadarajan P. Echocardiographic evaluation of patent foramen ovale and atrial septal defect. Echocardiography 2015;32 Suppl 2:S110-S124.

33. Kim YJ, Hur J, Shim CY, Lee HJ, Ha JW, Choe KO, et al. Patent foramen ovale: diagnosis with multidetector CT--comparison with transesophageal echocardiography. Radiology 2009;250:61-67.

34. Ferro JM. Cardioembolic stroke: an update. Lancet Neurol 2003;2:177-188.

35. Khetarpal V, Mahajan N, Madhavan R, Batra S, Mopala P, Sagar A, et al. Calcific aortic valve and spontaneous embolic stroke: a review of literature. J Neurol Sci 2009;287:32-35.

36. Clavel MA, Malouf J, Messika-Zeitoun D, Araoz PA, Michelena HI, Enriquez-Sarano M. Aortic valve area calculation in aortic stenosis by CT and Doppler echocardiography. JACC Cardiovasc Imaging 2015;8:248-257.

37. Larsen LH, Kofoed KF, Carstensen HG, Mejdahl MR, Andersen MJ, Kjaergaard J, et al. Aortic valve area assessed with 320-detector computed tomography: comparison with transthoracic echocardiography. Int J Car- 
diovasc Imaging 2014;30:165-173.

38. Pujadas R, Arboix A, Anguera N, Rafel J, Sagués F, Casañas R. Mitral annular calcification as a marker of complex aortic atheroma in patients with stroke of uncertain etiology. Echocardiography 2008;25:124-132.

39. Poh KK, Wood MJ, Cury RC. Prominent posterior mitral annular calcification causing embolic stroke and mimicking left atrial fibroma. Eur Heart
J 2007;28:2216.

40. Chen O, Dontineni N, Nahlawi G, Bhumireddy GP, Han SY, Katri Y, et al. Serial cardiac magnetic resonance imaging of a rapidly progressing liquefaction necrosis of mitral annulus calcification associated with embolic stroke. Circulation 2012;125:2792-2795. 